\title{
Afterthoughts from an environmental pollution discovery: Interview with Sören Jensen
}

\author{
This article belongs to Ambio's 50th Anniversary Collection. Theme: Environmental \\ contaminants
}

Sören Jensen

Published online: 19 January 2021

Q1: Your Ambio article about the discovery of PCBs as an environmental contaminant reads as a detective story that is made especially fascinating by all the threads that lead into and out of it. That said, were there still more tests you carried out that you in hindsight see as relevant?

A1: Maybe the mink (Neovison vison) experiment with DDT and PCB. Mats Olsson at the Swedish Royal Museum of Natural History collected mink from various parts of Sweden and I analyzed the DDT content and when PCB was identified that too. The concentrations were high for both DDT-metabolites and PCBs. That led to a mink experiment which Jan-Erik Kihlström at Uppsala University carried out. Minks were divided into four groups where one got PCB, another DDT, a third PCB+DDT added to the feed and a fourth was control. The females of the control and DDT groups each got an average of eight youths in the litter, while those who got PCB produced no offspring at all.

Q2: Those results I think pointed to the studies that your collaborator Mats Olsson made of Baltic seals. Can you tell a little about them?

A2: It had been noted for a while that the number of seals in the Baltic Sea declined. Fishermen, who carried out most of the observations, liked this development as they saw the seals as competitors for the fish, and as thieves and villains when they took fish out of the nets. They largely argued that the apparent decline in seals was due to migration, and pointed out that hardly any dead seals were found. The authorities anyhow reacted and limited seal hunting in Sweden, but the population decline continued. All three seal species, grey seal (Halichoerus grypus), harbor seal (Phoca vitulina) and ringed seal (Pusa hispida), were affected. Mats Olsson could document the decline, especially in the late 1960s and 1970s, and couple it to PCBs. He also saw that the uteri of females had occlusions and stenosis resulting in missed pregnancies and he noted other deleterious effects such as infected intestines and enlargements of adrenal glands in both sexes.

Q3: What were the greatest challenges in the identification of PCBs in environmental samples.

A3: Well, there were several. First was the question if the compound that caused the spikes in the chromatogram came from the sample or was present in the solvent or carrier gas. The gas flow was constant so if it had been the source the peaks would have been of constant height. Samples from the north of Sweden had lower ones than those from the south when the same amount of solvents were used so it had to be in the sample.

A mass spectrometer coupled to the gas chromatograph gave the atomic weight so it was just a matter of computing what combination and number of elements would give that number and assess which of those could form stable and persistent compounds, a lot of labor at the time but fully doable. Quantification was more complicated as there were no standards. The first solution I found gave reasonable figures, but perfect they were not.

Q4: Later, the knowledge that PCBs hade an even wider range of uses than those mentioned in the article, like in electrical transformers and certain types of printing ink, was reviled from users and producers and the application in hydraulic systems of military tanks and artillery, which are still not regulated, was found in war damage studies in the aftermaths of the Gulf War.

A4: Most OECD countries followed suit within a few years. Japan and USA dragged their feet with regulations of 
electrics applications but came on board within a decade. In the rest of the world not much happened for a longer time than that.

\section{Q5: And now?}

A5: I don't have any production figures but as concentrations found in environmental samples are lower today than they were in the 1970s more or less world-wide, so I guess PCB production and use has gone down too.

Q6: Besides the decline in PCBs, did the results of your work have even wider consequences?

A6: Well, I wrote in the article that most of the early concern about both chlorinated hydrocarbons and mercury came after applications in agriculture. There, the substances were used as poisons, mostly intended for insects and other organisms considered as pests, but many of them or their metabolites were subsequently found to affect also other species. With PCBs, we showed that persistent chlorinated hydrocarbons became environmental contaminants and caused ecological damage, also when their use was industrial and the property sought after was not toxicity. I think that became an important insight.

Q7: Your collaboration with Mats Olsson on PCBs in seals, and later with Arne Jernelöv on mercury, were early examples of analytical chemists collaborating with biologists to solve environmental problems. This now seems to be the rule rather than the exception. Is it so?
A7: It is clear that it was a fruitful combination and remains so. Today, scientific teams are often much larger and involve many more disciplines. Ours was a first step - a good one, but only a start in this respect.

Q8: What did the discovery of PCBs as environmental contaminants mean for yourself? You worked at the Department of Analytical Chemistry at the Stockholm University at the time but not in a leading position, and with the discovery you were propelled to world fame, at least in environmental circles.

A8: Well, it is true, more or less, but those circles were not so big at the time.

Q9: When you have been working with a problem for some time and finally think you have solved it, you often have a kick of euphoria and hybris. Did that happen to you when you realized that it was PCB?

A9: It did indeed. I was working late that night and I was jumping up and down on my own in the lab screaming with excitement before I run over to my professor's home. Widmark lived in a villa on the campus just by the lab. He had dinner guests but I just rushed in shouting "PCBs - it is PCBs" and hugging everyone.

Publisher's Note Springer Nature remains neutral with regard to jurisdictional claims in published maps and institutional affiliations.

Sören Jensen ( $\square)$

Address: Huddinge, Sweden. 